\title{
Embryos from in Vitro Maturation (IVM) Technique Can Be Successfully Vitrified Resulting in the Birth of a Healthy Child
}

\author{
Norma P. de Oliveira ${ }^{1}$, Caroline G. Dutra ${ }^{1}$, Gerta N. Frantz ${ }^{1}$, Carla G. Basso ${ }^{1}$, Marcela F. Fortis ${ }^{1}$, Nilo Frantz ${ }^{1}$ \\ ${ }^{1}$ Nilo Frantz Reproduction Center - Porto Alegre - RS - Brazil
}

\begin{abstract}
IVM can be an advantageous technique when applied to PCOS (Polycystic Ovarian Syndrome) patients. The oocytes are retrieved from antral follicles of non-stimulated ovaries, specially preventing hyperstimulation syndrome. Apart from its role as a reproductive treatment, IVM has emerged as a promising tool for emergency fertility preservation, since it can be performed flexibly in either follicular or luteal phase. A 34-year-old patient with PCOS, high body mass index and tubal factor was submitted twice to IVM treatment. Her husband has low count spermatozoa. The first IVM cycle was in 2009, she transferred 3 fresh embryos and got pregnant giving birth to a healthy boy weighing $3.3 \mathrm{~kg}$. In 2013, the patient returned for another IVM cycle and the embryos had to be vitrified because she failed to develop an adequate endometrium for transfer. In the next cycle, the endometrium was prepared using estrogen and progesterone and the two best embryos were warmed up and transferred. She became pregnant and after 36 weeks gave birth to a healthy girl weighing $2.7 \mathrm{~kg}$. She still has four embryos left to transfer. IVM may be an alternative technique to be considered when dealing with PCOS patients. Although clinical outcomes are currently inferior when compared with conventional hormone driven ART (Artificial Reproductive Techniques), it does apply in some cases while preventing hyperstimulation risks. Thus, embryos obtained by IVM can also be vitrified with successful outcomes.
\end{abstract}

Keywords: IVM, In vitro maturation, Embryos, Vitrification, Polycystic Ovarian Syndrome

\section{INTRODUCTION}

In vitro maturation (IVM) consists of aspirating immature oocytes from non-stimulated ovaries, and is an alternative treatment for women with Polycystic Ovarian Syndrome (PCOS). This technique can also be applied in patients not responding to conventional treatments, or patients with cancer who wish to cryopreserve their gametes, avoiding the risks brought about by hormonal stimulation.

It is estimated that over 4,000 babies have been born from this technique until 2014 (Gay parent magazine, 2014), since its first report in 1991(Cha et al., 1991). IVM offers some advantages over conventional treatments, especially because it avoids hormonal stimulation with high doses of gonadotropins and gonadotropin-releasing hormone $(\mathrm{GnRH})$ analogues. Its application minimizes the risk of Ovarian Hyperstimulation Syndrome (OHSS), a serious metabolic condition that can affect from 0.5 to $2 \%$ of women undergoing assisted reproduction techniques (ART) and up to $6 \%$ of patients having PCOS (MacDougall et al., 1993). Besides reducing treatment costs, IVM technique avoids uncomfortable injections of gonadotropins, which can be an advantage for some women.

IVM can be considered a viable and efficient alternative among assisted reproduction treatments (ART), respecting the indications for each patient.

The case presented below shows a successful application of IVM in two consecutive opportunities in a patient with PCOS and high body mass index (BMI 28.5). In the first IVM cycle, fresh embryos were transferred, and later, in a second cycle, the IVM embryos were vitrified and then transferred, in both cases, it produced healthy babies.

\section{CASE DESCRIPTION}

The patient is a 34-year old woman diagnosed with (PCOS), tubal and mild male factors. In 2009, she was submitted to the first IVM attempt. Follicular aspiration was carried out in a natural cycle before a dominant follicle developed. A dose of 10,000 IU of human chorionic gonadotropin (Choriomon-Meizler) was used as a trigger 36 hours before follicular puncture (Chian et al., 2000).

An experienced ultrasound physician performed aspiration of small follicles, with suction pressures between 80 and $90 \mathrm{mmHg}$. Follicular aspirates were filtered through a 70- $\mu \mathrm{m}$ nylon mesh cell strainer (BD Falcon, USA) and the cumulus oophorus complexes (COCs) were kept for three hours in pre maturation culture medium (Origio-Medicult IVM System). These procedures take longer work time and require greater attention than usual, given to the small size and morphology of immature oocytes often presenting a thick and compact layer of cumulus cells (Figure 1).

Subsequently, the oocytes were transferred to a maturation medium (Origo-Medicult IVM System) supplemented with recombinant FSH $100 \mu \mathrm{l}(0,075 \mathrm{IU} / \mathrm{mL}$ Gonal Merck), $10 \mu \mathrm{l}$ of hCG $(0.1 \mathrm{IU} / \mathrm{mL}$ - Chriomon Meizler) and $10 \%$ synthetic serum substitute (SSS, Irvine Scientific),

The oocytes were kept for $30 \mathrm{~h}$ in the maturation medium (Origio-Medicult System) and then were mechanically denuded. Twelve oocytes were collected and eight of them reached metaphase-II stage (maturation rate of $66.6 \%$ ). Insemination was carried out using

Figure 1. View of COCs on the day of follicular puncture for IVM. Note the more compact cumulus layers (A and $\mathrm{B}$ ) or more disperse ( $\mathrm{C}$ and $\mathrm{D}$ ) around the oocyte
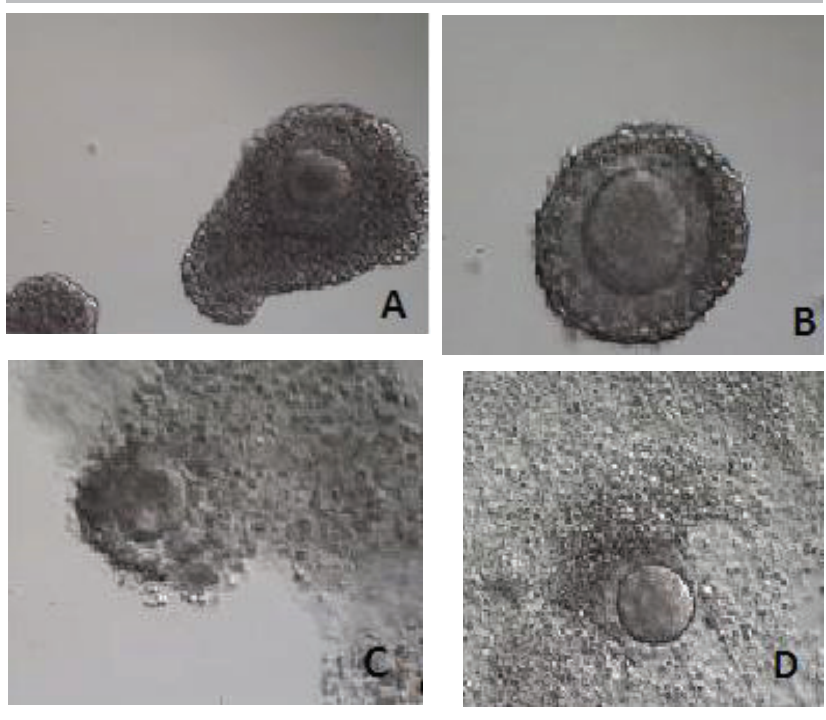
the intracytoplasmic sperm injection (ICSI) procedure with a fertilization rate of $50 \%$. After ICSI, the embryos were kept in conventional culture medium (LifeGlobal Media) supplemented with $10 \%$ synthetic serum substitute (SSS, Irvine Scientific) until the embryo transfer (ET). From the four embryos formed, three were selected for ET on day three (D3) and classified as 1-G1 (best grade) and 2-G4 (worst grade). A single fetus pregnancy resulted in the birth of a healthy baby weighing $3.3 \mathrm{Kg}$.

In 2013, the patient returned to the clinic and a new IVM cycle was indicated using the same previous protocol. Then we collected 10 COCs and kept them in maturation medium (Origio-Medicult System) for 30 hours. Six oocytes reached Metaphase II (MII), resulting in a maturation rate of $60 \%$. All ICSI inseminated oocytes fertilized and were classified at D3 as 1-G1, 2-G2, 1-G3 and 2-G4. The embryos were cryopreserved on D3, according to the regular vitrification protocol (Vitri-Ingá Ingamed; Almodin et al., 2010; Almodin \& Costa 2011; Almodin \& Costa 2014). All the embryos were vitrified because at that moment the endometrium was not adequate for transfer.

In the following cycle, the endometrium was supplemented with estradiol valerate, $6 \mathrm{mg}$, administered daily starting on the second day of the menstrual period (Primogyna-Bayer). For luteal phase support, when the endometrium reached $8 \mathrm{~mm}$ of thickness and a triple-line pattern, the patient received $800 \mathrm{~mm}$ of intravaginal progesterone (Utrogestan-Farmoquímica). The two best-classified vitrified embryos (G1 and G2) were then warmed up and transferred (Figure 2) After 12 days of transfer, beta-HCG was positive and pregnancy developed until 36 weeks with the birth of a healthy $2.7 \mathrm{Kg}$ girl. The patient still has four vitrified embryos.

Figure 2. IVM embryos at D3 transferred
after warming. A:G2 Embryo, B:G1 embryo
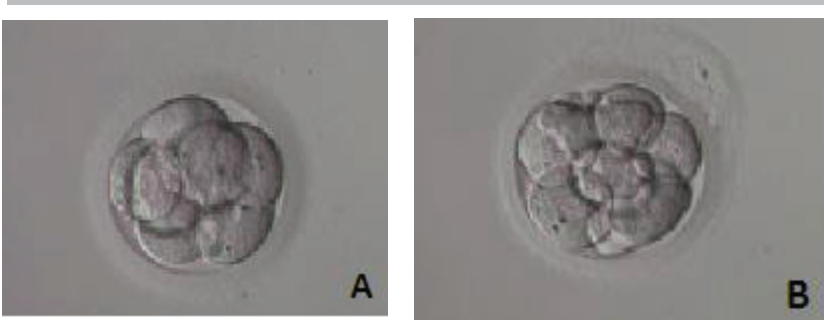

\section{DISCUSSION}

Despite not being a routine in most AR clinics, the IVM technique represents a viable and efficient tool for some patients, especially those having Polycystic Ovarian Syndrome.

The use of hCG trigger (Chriomon Meizler) by our team was based on the improvement of the oocyte nuclear maturation in vivo, although this mechanism is not very clear, since the diameter of aspirated follicles is smaller than $10 \mathrm{~mm}$ (Chian et al., 2000).

Jakimiuk et al. (2001) suggesting that POS patients may express luteinizing hormone (LH) receptors earlier in the granulose cells. The improved maturation of COCs with hCG could also be explained by the presence of LH in the theca cells, which could favor the expansion of cumulus cells, although this fact does not represent a significant difference in the potential of the formed embryo (Zheng et al., 2012).

Some authors have reported better maturation results between 28-32 hours of culture (Trounson et al., 2001, Jurema \& Nogueira 2006), and according to our experience, we opted for the 30-hour in the culture medium protocol (Origio-Medicult System), supplemented by recombinant
FSH (Merck S/A), hCG (Meizler), and $10 \%$ of exogenous protein (SSS Irvine). Considering the two referred trials, the average maturation rate was of $65 \%$, comparable to studies from most of the authors (Son et al., 2005, Walls et al., 2015).

ICSI insemination has been preferred in cases of IVM (Hwang et al., 2000) to show best fertilization rates when compared to conventional IVF. This difference is due to a possible zona pellucida hardening effect during the in vitro maturation process (Jurema \& Nogueira, 2006). In the described case, ICSI is also justified by the presence of the male factor. Considering the two IVM cycles described, the average fertilization rate was $75 \%$.

After fertilization, the embryo culture proceedings were the same as in routine cases, in this case using Global medium (LifeGlobal Media) supplemented with $10 \%$ synthetic serum substitute (SSS Irvine) until embryo transfer time at Day 3.

Vitrification has been a well-established adjuvant technique to conventional IVF, which can be also applied to embryos produced by in vitro matured oocytes (Son \& Tan, 2009; Ortega-Hrepich et al., 2013).

Although in this report the survival rate after warming up of 2 embryos was high, authors report lower survival rates in cases of IVM. The expected rate for traditional IVF cases would be around $90 \%$, while in cases of IVM one can expect survival rates of $70 \%$, after the warming stage. (Shalom-Paz et al., 2012).

This successful clinical case shows that IVM is a viable approach within the AR techniques offering lower cost, execution flexibility, good acceptance by patients, as well as an important tool to avoid the ovarian hyperstimulation syndrome. Vitrification of embryos produced by in vitro matured oocytes becomes also a promising alternative, enabling a most convenient occasion for embryo transfer, thus increasing pregnancy and birth rates.

Further studies must be developed for better understanding the follicular environment and its maturity, also for optimizing culture systems, so that results of this practice may be similar to conventional therapies. The spread of the IVM technique also requires investment in more detailed studies with larger number of cases and future monitoring of the baby's health.

\section{CONFLICT OF INTERESTS}

No conflict of interest have been declared.

\section{Corresponding author:}

Norma P. de Oliveira

Nilo Frantz Reproduction Center

Porto Alegre/ RS - Brazil

E-mail: norma@nilofrantz.com.br

\section{REFERENCES}

Almodin CG, Camara VCM, Paixão CL, Pereira PC. Embryo development and gestation using fresh and vitrified oocytes. Hum Reprod. 2010; 25:1192-8.

Almodin CG, Costa RR, eds. Criopreservação de gametas, embriões e tecido germinativo em laboratório de fertilização in vitro. Rio de Janeiro: Cultura Médica; 2011.

Almodin CG,Costa RR, eds. Criopreservação em Reprodução. Maringá: Dentalpress; 2014.

Cha KY, Koo JJ, Ko JJ, Choi DH, Han SY, Ion TK. Pregnancy after in vitro fertilization of human follicular oocytes collected from nonstimulated cycles their culture in vitro and their transfer in a donor oocyte program. Fertil Steril. 1991;55:109-13. 
Chian RC, Bucket WM, Tulandi T, Tan SL. Prospective randomized study of human chorionic gonadotrophin priming before immature oocyte retrieval from unstimulated women with polycystic ovarian syndrome.. Human Reprod 2000, 15:165-70.

Gay parent magazine. For gayparentmagazine One BrookIyn Couple, New Fertility Advancements Bring Double the Joy. Gay parent magazine. march-april 2014. available at: http://newayfertility.com/wp-content/uploads/2014/06/ GayParentsMagazine_March-April_2014_issue_93_13.9_ mb_download.pdf

Hwang JL, Lin YH, Tsai YL. In vitro maturation and fertilization of immature oocytes: a comprehensive study of fertilization techniques. J Assist Reprod Genet 2000; 17:39-43.

Jakimiuk AJ, Weitsman SR, Navab A, Magoffin DA. Luteinizing hormone receptor, steroidogenesis acute regulatory protein, and steroidogenic enzyme messenger ribonucleic acids are overexpressed in thecal and granulosa cells from polycystic ovaries.. J Clin Endocrinol Metab. 2001; $86: 1318-23$.

Jurema M, Nogueira D. In vitro maturation of human oocytes for assisted reproduction. Fert Steril. 2006; 86:1277-91.

MacDougall MJ, Tan SL, Balen A, Jacobs HS. A controlled study comparing patients with and without polycystic ovaries undergoing in vitro fertilization. Hum Reprod. 1993; 8:233-37.

Ortega-Hrepich C, Stoop D, Guzman L, Van Landuyt L,
Tournaye H, Smitz J, De Vos M. A freeze all stategy after in vitro maturation: a novel approach in women with polycystic ovary syndrome? Fertil Steril. 2013;100:1002-7.

Shalom-Paz E, Holzer H, Yang-Son W, Levin L, Tan SL, Almog B. PCOS patients can benefit from in vitro maturation (IVM) of oocytes. Eur J Obstetr Gynecol Reprod Biol. 2012; 165:52-6.

Son WY, Seok YL, Lim JH. Fertilization, Cleavage and blastocyst development according to the maturation timing of oocytes in in vitro maturation cycles. Hum Reprod. 2005; 20:3204-07.

Son WY, Tan SL. Comparison between slow freezing and vitrification for human embryos. Expert Rev Med Devices. 2009; 6:1-7.

Trounson A, Anderiesz C, Jones G. Maturation of human oocytes in vitro and their developmental competence. Reproduction. 2001; 121:51-75

Walls ML, Hunter T, Ryan JP, Keelan JA, Nathan E, Hart RJ. In vitro maturation as an alternative to standard in vitro fertilization for patients diagnosed with polycystic ovaries: a comparative analysis of fresh, frozen and cumulative cycle outcomes. Human Reprod. 2015; 30: 88-96.

Zheng X, Wang L, Zhen X, Lian Y, Liu P, Qiao J. Effect of hCG priming on embryonic development of immature oocytes collected from unstimulated women with polycystic ovarian syndrome. Reprod Biol Endocrinol. 2012;10:40 